\title{
¿Debe creer uno lo que supera la razón natural para salvarse?
}

\section{Comentarios sobre Santo Tomás en 1584 de P. de Aragón y de D. Báñez}

La relación entre la naturaleza y la gracia ha sido en todo momento objeto de interés por parte de la teología en los tiempos todos. Siempre se ha reconocido que el orden de la naturaleza es distinto del de la gracia. Y ha interesado en todo momento saber si esos dos órdenes, pese a ser distintos e independientes incluso, se hallan entre sí estrechamente relacionados, hasta el punto incluso de que no se agota en el primero el ser del hombre, pidiendo por tanto el primero la existencia del segundo; es decir, llamando el de la naturaleza al de la gracia.

Suele ser frecuente oponer en muchas ocasiones la fe a la gracia. Cuando se hace esto, ¿se está señalando que la fe pertenece a la naturale$\mathrm{za}$, sin caer en modo alguno dentro del campo de la gracia? Verdad es a este respecto que la fe se divide en natural y sobrenatural, adquirida o sobrenatural. La natural o adquirida es fruto del esfuerzo humano. La sobrenatural o infusa es gracia o regalo de Dios. El objeto de la adquirida es lo que está al alcance de las fuerzas naturales. El de la infusa supera tales fuerzas.

Desde esta distinción se cae en la cuenta de inmediato de que lo que se pregunta aquí es si se requiere creer la fe infusa o sobrenatural algo a lo que las fuerzas de la naturaleza: la fe natural o adquirida, son incapaces de alcanzar. Y es en este momento en el que se debe decir que esta obligación de creer no reside necesariamente en algo que añade Dios sobre lo existente naturalmente desde siempre, sino que tener que aceptar algo sobrenatural es algo connatural a la naturaleza misma, hasta el punto incluso de que todos pueden desde la sola naturaleza quedar obligados, y quedan de hecho, a aceptar lo que rebasa las fuerzas de ésta; es decir, algo que es gracia o regalo gratuito de Dios.

Pedro de Aragón ${ }^{1}$ y Domingo Báñez fueron dos teólogos salmantinos del siglo XVI. Al uno y al otro les une el haber dado a conocer mediante la

\footnotetext{
${ }^{1}$ Cf. JeRICó BeRmejo, I., La Escuela de Salamanca del siglo XVI. Una pequeña introducción. (Madrid 2005) 230-232

${ }^{2}$ Cf. Ibid. 220-224.
} 
imprenta en 1584 los comentarios a las primeras cuestiones de la Secunda Secundae que había compuesto Santo Tomás en el siglo XIII ${ }^{3}$. No hay razón aquí para extenderse en lo que supuso la Escuela de Salamanca en la teología de la Edad Moderna. Puede uno obtener información en otra parte al respecto 4 . Baste decir que los teólogos salmantinos del siglo XVI tomaron como maestro de la teología a Santo Tomás de Aquino, dejando orillado el tratamiento de las cuestiones de escuela, vía o corriente, en orden a concentrar todas sus fuerzas en exponer la teología que debía interesar a todos por ser de condición común. Y no hay duda de que esta problemática sobre si era necesario en todo momento creer algo que supera la razón natural.

\section{EXPOSICIÓN}

La misma se divide en dos partes. Se coloca en primer lugar lo dicho por el Aquinate en el siglo XIII. A continuación se pone lo expuesto en el XVI por Aragón y por Báñez.

\section{Santo Tomás de Aquino}

Comienza su exposición Santo Tomás sobre si es preciso creer algo superior a la naturaleza para obtener la salvación señalando que, al parecer, no es algo necesario, ya que parece que bastan para la salvación y la perfección de cualquier realidad las cosas que le convienen según su naturale$\mathrm{za}^{5}$. Ahora bien, como las cosas que son de fe exceden la razón natural del hombre al ser de lo que no aparece según ya se ha establecido, resulta que no parece que sea necesario creer para alcanzar la salvación ${ }^{6}$. De todas for-

3 Cf. Jericó Bermejo, I., La Escuela de Salamanca del siglo XVI. Una pequeña introducción. Madrid 2005.

4 " $L$ a secunda secundae, la más larga de todas las partes de la Summa, estaba terminada para la primavera de 1272". WeISPHEIPL J. A., Tomás de Aquino. Vida, obras y doctrina. (Pamplona 1994) 414.

5 "Ad tertium sic proceditur. $1^{\circ}$. Videtur quod credere aliquid supra rationem naturalem non sit necessarium ad salutem. Ad salutem enim et perfectionem cujuslibet rei ea sufficere videntur quae conveniunt ei secundum suam naturam". II-II, q. 2, a. 3, 1 um.

6 "Sed ea quae sunt fidei, excedunt naturalem hominis rationem, cum sint non apparentia, ut supra dictum est. Ergo credere non videtur necessarium esse ad salutem". II-II, q. 2, a. 3,1 um. 
mas, no se debe extraer sin más esta conclusión. Por el Nuevo Testamento hay constancia de que: "Sin fe es imposible agradar a Dios" (Heb 11,6)7.

Así las cosas, responde el Aquinate y dice que se encuentra en todas las naturalezas que concurren dos cosas a la perfección de una naturaleza inferior. Una de ellas es ciertamente lo que es según el movimiento propio, y que, en cambio, hay otra cosa que es según el movimiento de otra naturaleza superior. Así el agua es movida según el movimiento propio hacia el centro; pero, según el movimiento de la luna, se mueve alrededor del centro en conformidad con el flujo y el reflujo. De manera semejante, las órbitas de los planetas se mueven por los propios movimientos de Occidente a Oriente; pero se mueven por el movimiento de la primera órbita de Oriente a Occidente 8 .

Ahora bien, la naturaleza racional creada es la única que tiene una ordenación inmediata a Dios. Ello se debe a que las demás criaturas no alcanzan algo universal. Al contrario, se quedan sólo en algo particular, participando de la bondad divina, ya sea sólo en el ser, como las cosas inanimadas, ya sea también al vivir y conocer cosas singulares, como las plantas y los animales. Ahora bien, en cuanto conoce la razón universal de bien y de ser, tiene una ordenación inmediata al principio universal del ser ${ }^{9}$. ¿Qué debe decirse entonces como respuesta a lo que se ha objetado al principio? El Doctor Angélico señala a este respecto que se requiere en la naturaleza del hombre cierto conocimiento sobrenatural, por quedar ésta dependiente de una naturaleza superior y no bastar para su perfección el conocimiento natural ${ }^{10}$.

7 "Sed contra est quod dicitur Hebr. 11, vers. 6: Sine fide impossibile est placere Deo". IIII, q. 2 , a. 3 sed contra.

8 "Respondeo dicendum quod in omnibus naturis ordinatis invenitur quod ad perfectionem naturae inferioris duo concurrunt; unum quidem quod est secundum proprium motum; aliud autem quod est secundum motum superioris naturae, sicut aqua secundum motum proprium movetur ad centrum; secundum autem motum lunae movetur circa centrum secundum fluxum et refluxum. Similiter enim orbes planetarum moventur propriis motibus ab Occidente in Orientem, motu autem primi orbis ab Oriente in Occidentem". II-IiI q. 2, a. 3c.

9 "Sola autem natura rationalis creata habet immediatum ordinem ad Deum, quia caeterae creaturae non attingunt ad aliquid universale, sed solum ad aliquid particulare, participantes divinam bonitatem vel in essendo tantum, sicut inanimata, vel etiam in vivendo et cognoscendo singularia, sicut plantae et animalia. Natura autem rationalis, inquantum cognoscit universalem boni et entis rationem, habet immediatum ordinem ad univesale essendi principium". II-II, q. 2, a. 3c.

10 "Ad primum ergo dicendum, quod quia natura hominis dependet a superiori natura, ad ejus perfectionem non sufficit cognitio naturalis, sed requiritur quaedam supernaturalis, ut supra dictum est”. II-II, q. 2, a. 3 ad 1um. 


\section{Pedro de Aragón}

Santo Tomás sostuvo en la Suma Teológica y en el siglo XIII que se encuentran en todas las naturalezas ordenadas la concurrencia para la perfección de una naturaleza inferior dos realidades. Una de ellas es la que es según el movimiento propio. Ahora bien, es la otra la que tiene lugar según el movimiento de una naturaleza superior. Así, el agua queda movida al centro según el movimiento propio; pero queda movida según el movimiento de la luna alrededor del centro según el flujo y el reflujo ${ }^{11}$. Al respecto se pregunta Pedro de Aragón en el siglo XVI si es verdad esta afirmación. Es que es la tierra una a partir de las naturalezas inferiores, concurriendo a sü perfección únicamente el movimiento propio, que va precisamente hacia abajo en busca del interior del centro. Las realidades que se mueven a sí mismas hacen esto sólo por un único movimiento propio, el cual se desplaza de Oriente a Occidente. Tampoco se da en las realidades mixtas doble concurrencia alguna en orden obtener su perfección ${ }^{12}$.

¿Constituye entonces una falsedad decir que, en las naturalezas ordenadas todas, hay una concurrencia doble en orden a que los inferiores alcancen la perfección: la que es según el propio movimiento y la que es según un movimiento superior? ${ }^{13}$. Muchos son los que dicen por lo señalado que

11 "In omnibus naturis ordinatis inuenitur, quod ad perfectionem naturae inferioris duo concurrunt; unum quidem quod est secundum proprium motum; aliud autem quod est secundum motum superioris naturae. Sicut aqua secundum motum proprium mouetur ad centrum; secundum autem motum lunae nouetur circa centrum secundum fluxum et refluxum" II-II, q. 2, a. 3c.

12 "Circa primum fundamentum huius articuli est difficultas, vtrum verum sit? Et pro parte negatiua est argumentum: Nam terra est vna ex inferioribus naturis, et tamen ad suam perfectionem solum concurrit proprius motus, quo mouetur deorsum in centrum: similiter primum mobile tantum nouetur vnico proprio motu, scilicet, ab oriente in occidens: et in mixtis etiam non apparet, quomodo duo concurrant ad perfectionem illorum". A 2, III, 120a-120b.

A = ARAGÓN. P. DE, In II-II.

$2=\mathrm{q} .2$.

III $=$ a. 3 .

$120 \mathrm{a}-121 \mathrm{~b}=$ p. 120 , col. B-p. 121 , col. b.

Esta forma de citar se sigue a lo largo de las citas sobre P. de Aragón.

Éste es el título de la obra que aquí se utiliza: FRATRIS PETRI DE ARAGÓN, ORDINIS EREMITARUM SANCTI AUGUSTINI, ARTIUM ET SACRAE THEOLOGIAE MAGISTRI, ET IN CLARISIMA SALMANTICENSI ACADEMIA PUBLICI PROFESSORIS. In Secundam Secundae divi Thomae doctoris Angelici commentariorum. Tomus primus [...]. Salmanticae. Excudebat Joannes Ferdinandus. MDLXXXIIII.

13 "Ergo falsum est dicere, quod in omnibus naturis ordinatis, ad perfectionem inferioris concurrunt duo, vnum secundum proprium motum, et aliud secundum motum superioris". A 2, III, $120 \mathrm{~b}$. 
el fundamento colocado por Santo Tomás debe entenderse únicamente en las naturalezas que son de suyo partes que conservan el universo. Tal es el caso de los elementos y los cuerpos celestes, así como el de las criaturas intelectuales, poseedoras todas de ese movimiento doble, sin constituir esto una necesidad en la piedra y en la madera, como en otras semejantes, en orden a la conservación de la naturaleza. Quedaría entonces resuelta la objeción surgida desde las realidades mixtas ${ }^{14}$.

Francisco de Vitoria suministra según Pedro de Aragón una respuesta diferente al decir que las criaturas todas existentes en el mundo reciben alguna perfección desde las naturalezas superiores ya que influye el cielo en todo, dando esto como resultado que el fundamento de Santo Tomás es tenido también como verdadero en las realidades mixtas. Y así las cosas, se responde en el caso de la tierra que, en ella, hay una doble concurrencia también en orden a su perfección. Una de ellas se realiza en conformidad con el propio movimiento. Tal es el descansar en el centro. La otra es la iluminación del sol y la influencia de las estrellas en orden que, entre otras cosas, hace que aparezca en la tierra hierba que verdee y arboles frutales. Desde la Biblia se tiene constancia de que esto pertenece a la perfección de la tierra: "Así fueron acabados los cielos y la tierra, y todo su cortejo, etc." (Gén 2,1)15.

Queda según Santo Tomás inmediatamente ordenada con todo a un principio universal la naturaleza racional, en cuanto conoce la razón universal de lo bueno y de lo relativo a la entidad ${ }^{16}$. Y añade Pedro de Aragón que, respecto a esta primera razón del moverse, se contesta al lado de Cayetano, mostrando la posibilidad de hablar al respecto de dos maneras: teológica y filosóficamente. Si se habla de la primera manera, se halla el pri-

14 "Ad hanc difficultatem nonnulli dicunt, quod fundamentum D. Tho. est inteligendum tantum de illis naturis, quae per se sunt partes vniuersi conseruatiuae, qualia sunt elementa, et corpora coelestia, et creaturae intellectuales, quae omnia habent illum duplicem motum: at vero lapis et lignum, et alia huiusmodi non sunt necessaria ad conseruationem vniuersi: et per hoc soluitur instantia de mixtis". A 2, III, $120 \mathrm{~b}$.

15 "Caeterum Victoria aliter respondebat dicens, quod omnes creaturae in mundo existentes recipiunt a naturis superioribus aliquam perfectionem, nam coelum influit in omnia, ex quo fit, vt etiam in mixtis fundamentum $\mathrm{D}$. Tho. habeat verum. Et ad instantiam de terra respondetur, quod etiam ad illius perfectionem duo concurrunt: vnum secundum proprium motum, estque quies in centrum: alterum autem est illuminatio Solis, et influentia stellarum, ad hoc vt producat herbam virentem et lignum pomiferum, etc. Et quod hoc pertineat ad perfectionem terrae probatur ex illo Genes. c. 2. Igitur perfecti sunt coeli, et terra, et omnis ornatus eorum, etc.". A 2, III, 120 b.

16 "Natura autem rationalis, inquantum cognoscit universalem boni et entis rationem, habet immediatum ordinem ad universale principium". II-II, q. 2, a. 3c. 
mer cielo: llamado empíreo, lleno de espíritus: de ángeles y de bienaventurados. Por ellos, queda adornado en grado máximo y perfeccionado el hombre. Si se habla de la segunda manera, dice que se da en el primer ser hacia el que se mueve un movimiento circular que le es propio al hombre y que el carácter perpetuo de semejante movimiento provine de la inteligencia. Se da por tanto una doble concurrencia en orden a obtener el hombre su perfección ${ }^{17}$.

Y habla Pedro de Aragón de lo posible que es mostrar al respecto una conclusión en mejor sintonía con lo tenido en la mente por Santo Tomás. Se parte en ella de que éste intenta argumentar desde lo sensible y corporal hasta lo inteligible. Es como dice que, como el primero hacia el que se mueve obtiene el lugar primero entre lo corporal y lo sensible, no es precisa una doble concurrencia. Al fin y al cabo, no se halla subordinado a naturaleza superior corporal alguna. Al concurrir el mismo a la perfección de todos los otros cuerpos inferiores más allá de su propio movimiento, concurre también a la perfección de la criatura racional, por encima incluso del propio movimiento de ésta. Debe darse entonces la concurrencia de una operación divina ${ }^{18}$.

De todas formas, cuando Santo Tomás sostiene que la criatura racional está ordenada inmediatamente a Dios por conocer la misma la razón universal de lo bueno, es donde aparece la dificultad de si se está ante una verdad. Desde la enseñanza de Cayetano es preciso advertir pese a todo al respecto que puede considerarse a la naturaleza racional doblemente, según su ser y según su operación inmanente formada por el conocimiento y la avidez. Es posible establecer incluso una distinción ulterior; es decir, absolutamente y según una razón particular. Queda por cierto como algo claro que la criatura racional viene inmediatamente de Dios por creación según una

17 "Ad aliud autem de primo mobili respondetur cum Caietano in praesenti, quod de illo possumus loqui dupliciter, sicilicet, Theologice et Philosophice. Si loquamur primo modo, dicit, quod primum coelum, quod Empyreum appellatur, est plenum spriritibus Angelicis et beatis, quibus maxime ornatur, atque perficitur: si autem loquamur secundo modo, dicit, quod in primo mobili est motus circularis illi proprius, et praeterea est perpetuitas talis motus ab intellegentia proueniens: et sic ad illius perfectionem duo concurrunt". A 2, III, $120 \mathrm{~b}$.

18 "Vel secundo respondetur, et forsan melius, magisque ad mentem D. Tho. quod hic autor intendit arguere a sensibilibus, et corporalibus ad spiritualia, et intelligibilia, et dicit, quod quemadmodum inter corporalia et sensibilia primum locum tenet primum mobile, ad cuius perfectionem non est necesse, quod duo concurrant, quia non est subordinatum alteri naturae superiori corporali, cum tamen ipsum ad perfectionem omnium aliorum corporum inferiorum concurrat, vltra proprium motum ipsorum, sic etiam ad perfectionem creaturae rationalis, vltra proprium motum ipsius, debet concurrere operatio Dei". A 2, III, 121a. 
razón particular. También es cierto que, según el entendimiento y la voluntad, queda la criatura racional glorificada inmediatamente por Dios ${ }^{19}$.

Como esto no corresponde debatirlo aquí. Y con todo, se duda. Gira la misma sobre si la criatura racional según esta razón particular; es decir, en cuanto conoce la razón universal de bien y de ser, está ordenada inmediatamente a Dios como al principio universal del ser. A favor de la contestación negativa se coloca el argumento de que, si el hombre estuviera ordenado por este motivo inmediatamente a Dios como dice Santo Tomás, debería revelarse de inmediato a los hombres lo que es de fe; pero resulta que Dios lo hace mediante ángeles. Así queda claro desde la Sagrada Escritura y desde la doctrina de un libro atribuido Dionisio ${ }^{20}$ falsamente $^{21}$.

Aragón explica esta dificultad y dice al respecto que, aunque no se ordene el hombre inmediatamente a Dios al conocer la razón universal del bien y del ser, queda ordenado el mismo gracias a que los ángeles lo iluminan inmediatamente, convirtiéndolo en capaz de ordenarse a Dios, por conocer y apetecer el bien perfecto y universal. Se obtiene entonces la consecuencia de que se halla inmediatamente ordenado al bien mismo uni-

19 "Circa secundum fundamentum D. Tho. in quo dicit, quod creatura rationalis habet immediatum ordinem ad Deum, in quantum cognoscit vniuersalem rationem boni, est difficultas, de veritate illius? Ad cuius explicationem est aduertendum ex Caiet. in praesenti; quod natura rationalis potest dupliciter considerari: vno modo secundum suum esse; alio modo secundum operationem eius immanentem, quae est cognitio, et appetitio: et adhuc isto modo potest dupliciter considerari, scilicet, absolute, et secundum aliquam particularem rationem. Et quidem, quod creatura rationalis, secundum suum esse, sit immediate a Deo per creationem, est res manifesta. Quod autem, secundum intellectum et voluntatem, immediate beatificetur a Deo, etiam est certum". A 2, III, 121a.

20 "De todos los teólogos griegos, aquel que más influyó en Tomás y desde luego en todos sus contemporáneos fue el Pseudo-Dionisio Areopagita, pretendido discípulo de San Pablo, mencionado en los Hechos de los Apóstoles 17,34. El Corpus Areopageticum fue introducido en el occidente latino en el año 827, cuando el emperador griego Miguel Balbo envió una copia griega, consistente en cuatro tratados y diez cartas, a Luis el Piadoso, como muestra de amistad, [...] El autor del Corpus todavía es desconocido; parece haber vivido a principios del siglo VI; quizás fuera un monofisita, que trataba de pasar sus obras presentándose como contemporáneo de San Pablo. La supuesta antigüedad del Corpus le daba una autoridad solamente superada por los libros canónicos de la Escritura". WeISHEILPL, J. A., Tomás de Aquino. Vida, obras y doctrina. (Pamplona 1994) 210-211.

21 "Nec est praesentis loci hoc disputare. Sed quod vertitur in dubium est, vtrum creatura rationalis, secundum hanc particularem rationem, in quantum scilicet, cognoscit vniuersalem boni et entis rationem, habeat immediatum ordinem ad Deum, tamquam ad vniuersale essendi principium. Et pro parte negatiua est argumentum. Nam si homo hac ratione haberet immediatum ordinem ad Deum, vt D. Tho. dicit, sequeretur, quod Deus deberet immediate reuelare hominibus, quae sunt fidei, consequens autem est falsum: nam id facit mediantibus Angelis, vt patet ex Sacra Scriptura, et ex doctrina D. Dionysij de Ecclesiastica hierarchia, ergo". A 2, III, 121 a. 
versal: Dios, al conocer la razón universal de bien y de ser. Debe poseer en consecuencia una naturaleza capaz. Las cosas son así, a pesar de que se trate de algo remoto con conformidad con lo que toca al entendimiento y al apetito 22 .

Como se dicen las esferas inferiores poseedoras de una ordenación inmediata al primer ser que se mueve y queda ordenada también el agua a la luna por larga que sea entre ellas la distancia, lo mismo le ocurre a la criatura racional. Pese a la larga distancia que se da desde ella a la naturaleza divina, posee ella una ordenación inmediata como al objeto, fin y ser que la mueve. Sólo por Dios puede producirse el movimiento a tal objeto y a tal fin, el cual es el principio universal del ser. Esta misma razón existe también en la criatura angélica ${ }^{23}$.

\section{Domingo Báñez}

Ante sus ojos tiene muy presente Domingo Báñez el primer fundamento de Santo Tomás; es decir, que se encuentra en todas las naturalezas ordenadas la concurrencia para la perfección de una naturaleza dos realidades. Una de ellas es ciertamente la que es según el movimiento propio, siendo la otra pese a todo la que es según el movimiento de una naturaleza superior. Así, el agua queda movida al centro según el movimiento propio; pero queda movida según el movimiento de la luna alrededor del centro según el flujo y el reflujo. Al respecto dice Báñez que aparece un argumento contra esta exposición ${ }^{24}$. ¿Cuál es el mismo?

22 "Ad explicationem huius difficultatis dico, quod licet homo, in quantum cognoscit vniuersalem boni et entis rationem, non immediate ordinetur a Deo, sed mediantibus Angelis, a quibus immediate illuminatur, tamen, in quantum cognoscit bonum perfectum et vniuersale, et illud appetit, est illius capax atque ex consequenti, quantum ad hoc, habet immediatum ordinem ad ipsum bonum vniuersale, quod est Deus: quia enim cognoscit vniuersalem boni et entis rationem, quod etiam habeat naturam eius capacem, licet remote, secundum intellectiuam et appetitiuam partem, erit necesse". A 2, III, 121a.

23 "Vnde quemadmodum inferiores spherae dicuntur habere immediatum ordinem ad primum mobile, et aqua ad Lunam, quanuis haec inter se multum distent: ita etiam creatura rationalis, quanuis multum distet a natura diuina, in quantum tamen est ipsius vniuersalis boni capax, habet immediatum ordinem ad illum, vt ad suum obiectum, et finem, et mouens. Nihil enim potest mouere ad tale obiectum, et ad talem finem, nisi solus Deus, qui est vniuersale principium essendi. Et eadem ratio est de natura Angelica”. A 2, III, 121a-121b.

24 "Circa hunc articulum arguitur primo contra primum fundamentum Diui Thomae, scilicet quod in omnibus naturis ordinatis inuenitur, quod ad perfectionem naturae duo concurrunt. Vnum quidem quod est secundum propium motum: aliud autem quod est secundum 
Se aduce en contra que la esfera primera de las órbitas celestes no queda perfeccionada por naturaleza alguna superior, resultando de ello entonces que el fundamento colocado aquí carece de alcance universal. Un argumento semejante a éste se extrae de la tierra, la cual no recibe al parecer movimiento alguno de naturaleza alguna superior a la que quede subordinada. Lo que es mezcla de cielo y tierra no posee perfección alguna o movimiento comunicativo venido de una naturaleza superior, siendo entonces esto lo que convierte en falso el fundamento puesto ${ }^{25}$.

Recuerda Báñez asimismo que colocaba Santo Tomás al respecto un segundo fundamento, al decir que la criatura racional se halla ordenada inmediatamente a Dios, surgiendo en contra de esto el argumento de que los ángeles iluminan a los hombres y que, incluso, los ángeles superiores iluminan a los inferiores, tal como quedó escrito en un libro del PseudoDionisio. Por otra parte, el mismo Santo Tomás enseña que la revelación divina llega hasta los inferiores con un cierto orden desde los superiores, debiendo admitirse entonces que toda criatura racional no guarda por tanto un orden inmediato a Dios, dándose esto sólo en la criatura suprema ${ }^{26}$.

Sería posible decir además entonces aquí que se tiene la impresión por la razón desde los dos fundamentos colocados por el Aquinate que se sigue solamente de ello que el hombre es movido por dos motivos según el orden de la naturaleza; pero que no existe exigencia de que sea movido por cier-

motum superioris naturae. Sicut aqua secundum motum propium mouetur ad centrum: secundum autem motum Lunae nouetur circa centrum secundum fluxum et refluxum". B 2, III, 393. B = BÁÑEZ, D.., In II-II.

$2=\mathrm{q} .2$.

III $=$ a. 3 .

$393=$ p. 393 .

Esta forma de citar se sigue a lo largo de las citas sobre D. Báñez.

Éste es el título de la obra que aquí se utiliza: AUTORE FRATRE DOMINICO BAÑES [...], De Fide, Spe et Charitate [...] scholastica commentaria in secundam secundae Angelici Doctoris partem, quae ad quaestionem quadragessimam sextam protenduntur [...] Salmanticae. Apud S. Stepahnum Ordinis Praedicatorum MDLXXXIIII.

25 "Est argumentum. Nam prima sphera orbium coelestium non perficitur ab aliqua natura superiori, ergo fundamentum non est vniuersale. Simile argumentum possumus colligere de terra; quae non videtur recipere aliquem motum a superiori natura, cui subbordinatur. Mixta enim nullam habent perfectionem aut motum communicatum a superiori natura, ergo falsum est fundamentum". B 2, III, 393.

26 "Secundo arguitur contra secundum fundamentum, scilicet, quod creatura rationalis habet immediatum ordinem ad Deum. Nam angeli illuminant homines: imo et angeli superiores illuminant inferiores, sicut docet Diuus Dyonis. Capit. 12. Caelestis hierarc. Et Diuus Thomas art. 6. huius quaestionis docet, quod diuina reuelatio ordine quodam ad inferiores peruenit per superiores, ergo non omnis cratura rationalis habet immediatum ordinem ad Deum sed tantum suprema creatura". B 2, III, 393. 
to movimiento sobrenatural de fe. Pero reconocer que es con todo verdad el primer fundamento si se miran los movimientos desde el orden de la naturaleza ${ }^{27}$. Y esto se confirmaría además porque, si se quiere extraer desde tales fundamentos eficazmente la conclusión de que el hombre es movido desde la fe, se deduciría también que se presentó claramente Dios como el fin natural del hombre, lo cual niegan muchos de los tomistas, y que es además lo que parece sostener también Santo Tomás 28 .

Por otra parte, habría que contar con que el hombre debe ser perfecto en cuanto a su naturaleza, requiriéndose para que alcance su perfección el doble movimiento: el propio y el comunicado por una naturaleza superior. Habría que admitir en consecuencia entonces que el hombre requiere naturalmente esa doble operación: la propia y la comunicada por una naturaleza superior ${ }^{29}$. Pero da la casualidad de que esto se opone también a lo dicho por Santo Tomás de que se realiza la comunicación desde una naturaleza superior sucesivamente a la manera de aprendizaje. Los niños bautizados que mueren antes del uso de razón llegan a la visión de Dios sin disciplina alguna. Y Cristo el Señor recibió firmemente la claridad de la visión de Dios al mismo tiempo y de manera instantánea en cuanto hombre. No hubo necesidad de que la recibiera sucesivamente ${ }^{30}$.

A esto dirá Báñez que, si se considera la primera esfera celeste físicamente, como el primer ser que se mueve, ha de decirse que recibe ésta gran perfección la misma desde el ángel que la mueve ${ }^{31}$. Gracias a la fuerza comunicada por el ángel da la misma lugar a la vida en los que son inferio-

\footnotetext{
27 "Arguitur tertio contra consequentia rationis. Nam ex illis duobus fundamentis solum videtur sequi, quod homo moueatur duobus motibus secundum ordinem naturae, non autem quod moueatur motu quodam supernaturali fidei: nam primum fundamentum de motibus secundum ordinem naturae verum habet". B 2, III, 394.

28 "Et confirmatur. Nam si ex illis fundamentis volumus colligere efficciter, quod homo moueatur motu fidei, etiam colligemus, quod Deus clare visus est finis naturalis hominis, quod plures Thomistarum negant, et Diuus Thomas 1. part. quaest. 12. id videtur asserere". B 2, III, 394.

29 "Probatur sequela. Nam homo debet esse perfectus secundum naturam, igitur si quae libet res ad suam perfectionem requirit duplicem motum, vnum propium alterum communicatum a superiori natura, sequitur etiam, quod homo naturaliter requirit duplicem illam operationem, vnam propriam alteram communicatam a superiori natura". B 2, III, 394.

30 "Quarto argumentor contra illud, quod ait Diuus Thomas, quod ita communicatio a superiori natura sit per modum addiscentis successiue. Pueri enim baptizati discedentes ante vsum rationis perueniunt ad visionem Dei sine aliqua disciplina succesiua. Et firmiter Christus Dominus in quantum homo non succesiue sed simul statim recipit claram Dei visionem". B 2, III, 394.

31 "Ad argumenta sic dicendum est. Ad primum argumentum respondetur, quod si prima sphera caelestis physice consideretur pro primo mobili, magnam perfectionem recipit ab angelo mouente". B 2, III, 394.
} 
res. No tiene la vida en sí. Ahora bien, no debe olvidarse que recibe la perfección la primera esfera celeste también desde un círculo superior llamado por los teólogos el cielo empíreo, adquiriendo por el mismo constancia cierta de contener todo y de producir reposo cierto en los inferiores. El cielo empíreo es descanso en grado máximo. Báñez remite en este punto a leer la exposición de Santo Tomás ${ }^{32}$.

Ahora bien, si se toma la esfera primera por el cielo empíreo, añade Báñez que se dirá que participa asimismo de la máxima perfección gracias a los ángeles, los cuales existen y comunican cierta fuerza e, incluso, la causalidad. La tierra recibe asimismo desde las naturalezas superiores una perfección grande, como es la de dar lugar a las plantas y al oro. Las realidades mixtas compuestas de cielo y tierra reciben asimismo cierta fuerza venida de más alto. Lo enseña por lo demás Santo Tomás al señalar cómo posee el imán la fuerza de atraer el hierro mediante la impresión del cuerpo celeste; es más, gracias a tal impresión hace que, si el hierro se colocara en equilibrio, fuera movido hacia el polo ${ }^{33}$. Cayetano dice a este respecto que habla Santo Tomás de los cuerpos simples que son partes principales del universo y no se corrompen ${ }^{34}$.

Como enseñó el Aquinate, es Dios el único que mueve como causa eficiente el entendimiento y la voluntad de los hombres; pero enseñó que es el ángel y también el hombre quien, al proponer el objeto y colocar la luz, ilumina. Es más, un ángel ilumina a otro ángel a la manera de causa moral o artificial. Ésta presupone siempre una naturaleza determinada al fin ${ }^{35}$. Asi-

32 "Nam ex virtute angeli sibi communicata vitam efficit in his inferioribus, cum tamen illam in se non habeat. Recipit etiam perfectionem a superiore orbe, qui a Theologis dicitur caelum empyreum, a quo habet constantiam quandam continendi omnia et efficiendi quietem quandam in his inferioribus, quoniam coelum empyreum maxime quiescit. De qua re videatur Diuus Thomas 1. part. quaest. 66. artic. 3. ad secundum". B 2, III, 394.

33 "Si autem prima sphera accipiatur por caelo empireo, tunc dicimus, quod etiam participat perfectionem maximam $a b$ angelis existentibus et communicantibus quandam virtutem et etiam participat perfectionem maximam ab angelis existentibus et communicantibus quandam virtutem et etiam causalitatem. Caeterum terra etiam recipit a naturis superioribus perfectionem magnam, scilicet, virtutem germinandi plantas et aurum. Mixta denique etiam recipiunt quandam virtutem a superioribus, vt Diuus Thomas docet de veri. auast. 22. artic. 13. ubi ait, quod magnes habet virtutem attractiuam ferri ex impressione corporis caelestis: imo etiam ex eadem impressione habet quod moveat ferrum in equilibrio positum versus polum". B 2, III, 394.

34 "Sed Caietanus in hoc loco dicit, Diuum Thomam loqui de corporibus simplicibus, quae sunt partes principales vniuersi, quae quidem non corrumpuntur". B 2, III, 394.

35 "Ad secundum argumentum respondetur, quod vt Diuus Thomas docuit optime 1. 2. quast. 9. artic. 6. solus Deus mouet vt causa efficiens intellectum et voluntatem hominis. Nihilominus proponendo obiectum et illuminando hominem illuminat angelus et etiam homo hominem: quin etiam et vnus angelus alium illuminat ad modum causae moralis vel artificialis, quae praesupponit semper naturam determinatam in ordine ad finem". B 2, III, 394. 
mismo, se puede decir también aquí a modo de explicación más amplia desde la solución suministrada que el mantenimiento de un orden inmediato a Dios consiste en poseer inclinación inmediata al bien en general, lo cual surge precisamente de que la sola criatura racional conoce el bien universal. Tal inclinación es adecuada como resultado al conocimiento. Tiende por tanto al bien universal y común ${ }^{36}$.

Una criatura racional o intelectual más perfecta que otra es la que ilumina y perfecciona a otra criatura racional sobre algunos medios para lograr el fin. Esto nada quita por lo demás a esa criatura racional inferior en orden a mantener un orden inmediato a Dios como al fin. Y así es como ha de entenderse a Santo Tomás cuando enseña que no quedan obligados todos por igual a la posesión de la fe explícita, siendo precisamente los mayores y los primates en el oficio y en la dignidad eclesiástica quienes deben poseer una fe explícita que cumpla e ilumine a los inferiores. Ciertamente, esto queda entendido en relación a la explicación de lo que se cree. No se entiende de la infusión de la luz de la fe. Allí queda todo hombre iluminado inmediatamente por Dios y por el Espíritu Santo ${ }^{37}$.

La consecuencia expuesta Santo Tomás debe entenderse desde lo que es menos a lo que más, desde la suposición de que Dios hubiera ordenado al hombre al fin sobrenatural, que es precisamente lo enseñado por la fe. De esta manera discurre el argumento y llega el mismo a la consecuencia. Las otras realidades naturales lo precisan en orden a ser perfeccionadas en sus naturalezas y a alcanzar la perfección sobrenatural. No se sigue entonces de lo anterior que el fin natural del hombre fuera la visión beatífica ${ }^{38}$.

\footnotetext{
36 "Secundo respondetur et explicatur amplius haec solutio, quod habere immediatum ordinem ad Deum est habere inmediatam inclinationem ad bonum in communi: quod quidem oritur ex eo, quod sola creatura rationalis cognoscit vniuersale bonum. Vnde eiusmodi inclinatio est adaequata cognitioni: et ideo tendit in bonum in vniuersali et in communi". B 2, III, 394-395.

37 "Cum hoc tamen stat, quod aliqua creatura rationabilis vel intellectualis, quae perfectior est, illuminet et perficiat alteram creaturam rationalem circa quaedam media ad finem. Sed hoc non tollit, quominus creatura illa rationalis inferior habeat inmediatum ordinem ad Deum tanquam ad finem. Et ita intelligendus est $D$. Thomas in art. 6 . Vbi docet, non omnes aequaliter teneri a habendam fidem explicitam; sed qui sunt maiores et primates in officio et dignitate Ecclesiastica debent habere fidem explicitam, vt perficiant et illuminent inferiores. Quod quidem intelligitur quantum ad explicationem eorum quae creduntur, non autem quantum ad infusionem luminis fidei: nam quantum ad hoc omnis homo illuminatur immediate a Deo et ab Spiritu sancto". B 2, III, 395.

38 "Ad tertium argumentum respondetur, consequentiam $\mathrm{D}$. Thomae tenere a minore ad maius, supposito quod Deus ordinaverit hominem ad finem supernaturalem, vt docet fides. Tunc enim procedit argumentum. Bene namque sequitur; aliae res naturales indigent, ad hoc quod perficiantur in suis naturis, et consequatur perfectionem supernaturalem. Vnde ex hac ratione non sequitur, quod finis naturalis hominis sit visio beatifica". B 2, III, 395.
} 
Dicen algunos que los párvulos creen, tanto por el hábito de la fe como gracias a un acto ajeno, así como que basta esto para que sea verdad la proposición de Santo Tomás. Aquí se dirá, señala Báñez, que se expresa mejor en este terreno Cayetano al decir que el Aquinate está hablando de los adultos poseedores de actos propios, quedando inscrita entonces esta cuestión sobre el acto de fe sin basarse en el hábito. Lo objetado sobre Cristo el Señor no atenta en contra de la opinión de Santo Tomás. Es que habla éste de hombres que son puros hombres y deben llegar por el propio acto al último fin. Cristo lo consiguió por la unión personal con la humanidad. Ese fin natural se da entonces en Cristo en razón del sujeto ${ }^{39}$.

\section{CONCLUSIÓN}

¿Cuál es la fe que obliga en orden a alcanzar la salvación? A esto ha de responderse contundentemente que no es la adquirida o natural, sino la infusa o sobrenatural. Y objetivamente, eso que se ha de creer para obtener la salvación se halla más allá de la razón natural y ésta queda obligada a aceptarlo. Ciertamente, no ha de acogerlo desde la sola naturaleza racional, ya que de hecho lo rebasa; pero está al menos ante algo que no debe rechazar. La razón humana ha de admitir que es razonable que se adhiera a lo que le supera. Esto es algo que no se debe olvidar.

Parte de las cosas que se aceptan desde la fe sobrenatural e infusa se hallan con todo al alcance de la razón natural. Hay otras cosas pese a todo que nunca las habría barruntado siquiera la sola razón natural si no se le hubieran propuesto desde una revelación gratuita y extraordinaria. Esas verdades son los artículos de fe, entendidos en el sentido estricto; es decir, como verdades especialmente difíciles de ser vistas por el entendimiento humano. Aquí puede verse lo que dice el Aquinate en el artículo sexto de la cuestión sexta de la Secunda Secundae.

39 "Ad quartum argumentum aliqui dicunt, quod paruli credunt, et per habitum fidei et per actum alienum. Et hoc sufficit ad veritatem propositionis D. Thomae. Sed melius ait Caietanus in hoc articulo, D. Thomam hic loqui de adultis, qui habent proprios actus. Etenim quaestio haec inscribitur de actu fidei, non de habitu. Quod autem in eodem argumento objicitur de Christo domino, nihil facit contra sententiam D. Thomae. Nam ille loquitur de hominibus, qui sunt puri homines et per proprium actum debent peruenire ad vltimum finem: Christus autem assequutus est illum ex vnione personali ad humanitatem; quare finis ille naturalis est Christo ex ratione suppositi". B 2, III, 395. 
Una cosa es por supuesto decir que todos los artículos de fe han de ser creídos por todos con fe infusa para obtener la salvación y otra muy distinta decir que han de saberse estos artículos todos expresamente. Es claro que nada puede creerse si existe en sí como tales. Ante la vista de los hombres aparecieron los artículos con la revelación personal de Cristo. Desde entonces son los mismos obligatorios para todos. ¿Qué obligación tenían entonces los hombres de creer si querían alcanzar la salvación? Todos debían creer los artículos reducidos en las dos verdades básicas de la existencia de Dios y de la remuneración universal (Heb 11,6).

$Y$ a este respecto hay que señalar que, si se encuentran dentro de estas dos verdades básicas todos los artículos de la fe en forma reducida, eso significa reconocer que esas dos verdades no son en cuanto a contenido objetivo las que el filósofo o cualquier hombre ateniéndose sólo a las fuerzas de la naturaleza es capaz de creer. Se trata de una existencia y remuneración divinas que sobrepasa lo que es capaz de captar de suyo y de aceptar de suyo el hombre con las solas fuerzas naturales desde la sola razón.

Santo Tomás expuso ciertamente en el siglo XIII desde dos fundamentos y de forma muy sencilla cómo todo lo creado: lo inanimado, lo inanimado y los hombres, guardan una relación con una realidad superior; pero añadiendo que el hombre es el único que se halla ordenado directamente a Dios. El fin definitivo del hombre no es una naturaleza material o espiritual creada más o menos perfecta. Al hombre le colma definitivamente Dios solo.

Es desconocer esta condición real del hombre tratar de rebajar su altura y destino acoplándolo exclusivamente con los seres inanimados o animados, fueran éstos vegetales o animales. Tampoco se halla el hombre supeditado a seres más perfectos que él, como son los ángeles. Éstos cumplen encargos de Dios ciertamente; pero no se imponen a los hombres por su superioridad natural, sino por ser encargados por Dios de realizar determinadas tareas en los hombres. Admitir esto no significa en modo alguno tener que aceptar que los ángeles, criaturas sólo espirituales, son meta a la que deben dirigirse los humanos en orden a su perfección.

Por otra parte, aunque el hombre es naturaleza creada, como lo son los seres inanimados y animados: casos de los vegetales y de los animales, no queda el mismo absolutamente condicionado a alcanzar unos objetivos meramente naturales en cuanto a la finalidad de su misma naturaleza; es decir, no tiene por qué alcanzar siempre primero una meta natural para poder desde ella elevarse hacia la meta definitiva que es Dios.

Asimismo, en modo alguno debe entenderse que lo sobrenatural obliga sólo al hombre en la medida en la que se convierte en natural. Queda obli- 
gado el hombre desde lo sobrenatural desde el primer momento y es para él algo a lo que no debe renunciar; es decir, nunca debe eximirse de la exigencia constante de lo sobrenatural. Dijo Santo Tomás: "Por tanto, la perfección de la criatura racional no consiste solamente en lo que le compete según su naturaleza, sino también en lo que le atribuye desde cierta participación sobrenatural ${ }^{40}$.

Ignacio JERICó BERMEJO

40 "Perfectio ergo rationalis creaturae non solum consistit in eo quod ei competit secundum suam naturam, sed in eo etiam quod ei attribuitur ex quadam supernaturali participatione". II-II, q. 2, a. 3,c. 